\title{
Applications industrielles des lasers U.V. au Centre Commun de Recherches Louis-Blériot d'AEROSPATIALE
}

\author{
B. Esmiller et V. Baudinaud
}

AEROSPATIALE, Centre Commun de Recherches Louis-Blériot, 12 rue Pasteur, 92152 Suresnes cedex, France

\begin{abstract}
This paper concerns investigations conducted by AEROSPATIALE (Louis-Blériot Joint Research Center) on UV radiation-matter interaction and on the potential industrial applications like laser paint stripping, machining of special materials (ceramics, composites,) and the surface annealing of metals.
\end{abstract}

\section{INTRODUCTION}

Le développement récent de nouvelles sources laser émettant dans l'ultraviolet comme le laser excimère a permis d'envisager de nouvelles applications industrielles des lasers, en particulier dans l'aéronautique. Ce résumé présente donc les activités du Centre Commun de Recherches Louis Blériot d'AEROSPATIALE sur le thème laser excimère. Les travaux réalisés à l'AEROSPATIALE ont permis d'une part de comprendre les mécanismes d'interaction laser UV-matériau et d'autre part d'évaluer les potentialités des lasers excimères pour différentes applications afin de développer de nouveaux procédés industriels pour l'aéronautique tels que l'usinage de matériaux composites, le traitement de surface et le décapage de peintures.

\section{USINAGE DE MATÉRIAUX COMPOSITES}

\subsection{Etudes amont}

Afin de mieux appréhender la phénoménologie de l'interaction laser UV-matériau, nous avons réalisé différentes expériences, sur une céramique massive (carbure de silicium) et un métal (aluminium), telles que :

- des mesures de reflectivité, de pression et de rugosité

- l'imagerie de la vapeur ou du plasma

- des analyses chimiques de surface afin d'évaluer les changements de composition des matériaux après une irradiation laser

- la mesure du taux d'ablation (voir figure 1) et de rendement d'ablation

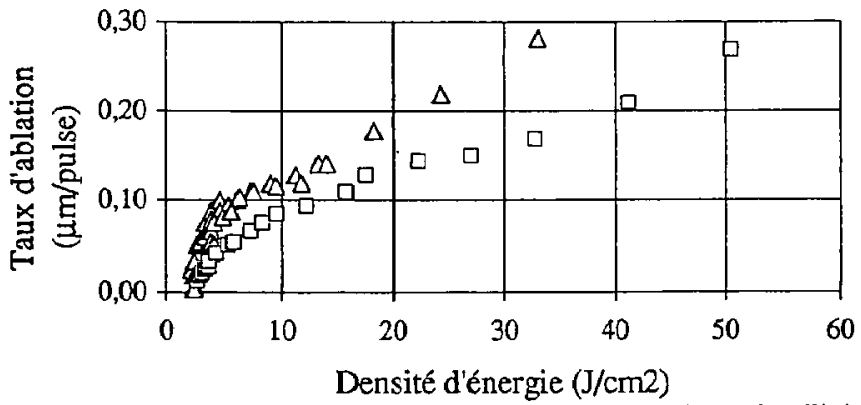

$\triangle \mathrm{XeCl}(308 \mathrm{~nm})$

$\square \operatorname{KrF}(248 \mathrm{~nm})$

Figure 1 : Courbes d'ablation du carbure de silicium 


\subsection{Applications industrielles}

Les différents essais de découpe ou de perçage ont montré une amélioration des qualités et des temps d'usinage de matériaux composites ( $\mathrm{SiC} / \mathrm{MAS}, \mathrm{SiC} / \mathrm{SiAlON}$, carbone/carbone) difficiles, voire impossibles, à usiner par des procédés conventionnels.

\section{REFORMATION DE SURFACES PAR LASER}

L'étude de l'action de nouveaux lasers sur la reformation de surface de matériaux s'inscrit dans le cadre d'un programme de recherche subventionné par le M.R.E.S. regroupant la Direction des Méthodes et Moyens Industriels de PSA a Meudon, SYLAREC (Centre Commun de Recherches Louis Blériot d'AEROSPATIALE à Suresnes et CEA Saclay), l'Institut de Mécanique des Fluides de Marseille et le centre des Matériaux de l'Ecole Nationale superieure des Mines de Paris.

L'étude repose sur la détermination des possibilités d'applications de nouveaux lasers (excimère et à vapeur de cuivre) pour operations de traitements de surfaces de pièces aéronautiques et automobiles.

Les objectifs du traitement avec ce type de laser a bas flux sont :

- modification des critères d'état de surface (rugosité)

- amélioration des proprietés superficielles par recristallisation voire amorphisation de surfaces (résistance a la corrosion, durcissement)

- amélioration des propriétés tribologiques voire mécaniques (fatigue).

En particulier dans le cas de la fonte, le désenclavement des nodules de graphite après usinage afin de lui rendre son rôle de lubrifiant et l'élimination des particules susceptibles de se détacher de la pièce en service et de provoquer des grippages permettent d'augmenter d'un facteur 10 à 50 la résistance à l'usure pour des fluences supérieures à $2 \mathrm{~J} / \mathrm{cm}^{2}$ et un seul tir laser.

Pour les céramiques, et plus particulièrement le carbure de silicium (SiC), la refusion ou la "cicatrisation" de la surface par laser améliorent son comportement thermique en réduisant, voire en supprimant sur les zones traitées, les porosités superficielles induites par le frittage des éprouvettes. Cela est rendu possible par la fusion du matériau lors de l'irradiation, et ceci au dessus d'une fluence caractéristique du laser. Cette étude a mis en relief l'existence d'une fluence seuil $\left(7 \mathrm{~J} / \mathrm{cm}^{2}\right)$, au-delà de laquelle de nouvelles porosités superficielles apparaissent. Cependant les traitements laser n'améliorent pas sensiblement les propriétés mécaniques du $\mathrm{SiC}$ en raison de certaines ses caractéristiques (défauts intemes pilotant probablement les mécanismes de rupture).

\section{DÉCAPAGE DE PEINTURES}

Les objectifs de ces travaux sont d'obtenir un décapage sélectif de peintures et d'atteindre des rendements de décapage supérieurs à $10 \mathrm{~m}^{2} /$ heure.

Les premiers essais de décapage de peintures aéronautiques sur matériaux composites ont permis d'évaluer les rendements obtenus par laser excimère $\left(0,5\right.$ a $0,8 \mathrm{~m}^{2} /$ heure pour un laser de $80 \mathrm{~W}$ ) et les effets sur le composite puis de comparer avec différentes sources laser disponibles au Centre Commun de Recherches Louis-Blériot d'AEROSPATIALE : laser YAG déclenché $(30 \mathrm{~W})$, laser YAG relaxé $(900 \mathrm{~W})$ ou laser $\mathrm{CO}_{2}$-TEA $(60 \mathrm{~W})$

Les résultats obtenus sont également comparés avec les procédés de décapage par projection de médiaplastiques ou amylacés, de décapage cryogénique ou par jet d'eau. 